\title{
Applying LSTM to Enable Cache Prefetching to Optimize Flow Table Update Efficiency in SDN Switches
}

\author{
Liangding Li \\ University of Central Florida \\ ECE Department \\ Orlando, FL, USA \\ leoriohope@knights.ucf.edu
}

\author{
Jiapeng Chi \\ University of Central Florida \\ ECE Department \\ Orlando, FL, USA \\ jiapengchi@knights.ucf.edu
}

\author{
Jun Wang \\ University of Central Florida \\ ECE Department \\ Orlando, FL, USA \\ Jun.Wang@ucf.edu
}

\begin{abstract}
Due to the fast growth of the Internet and social network, a massive amount of data has been generated and move to the cloud. With the ability to separate the control and data plane, SDN (Software Defined Network) provide an emerging solution for data transportation management tasks in the data center. In recent years, more literature focused on using SDN to manage data center network. It has been shown that SDN switch can support fine-grained rule matching with more than 12-tuple flow. However, Ternary Content Addressable Memory (TCAM), which used to store the flow table in SDN switch, has limited capacity and power-hungry. The performance of the data center throughput would reduce dramatically due to flow table overflow. Previous literature proposed two kinds of solutions, rule replacement and rule caching. In this paper, we propose a new rule caching method based on Long short-term memory (LSTM) to improve the cache hit ratio in SDN switches. From the experiment result, we surprisingly find that the deep learning based prefetching model can predict future flow rules with high accuracy. And then improve the cache hit ratio on TCAM compare with the famous FIFO and LRU cache.
\end{abstract}

\section{CCS Concepts}

- Networks $\rightarrow$ Bridges and $S$ witches

\section{Keywords}

SDN; flow table; LSTM; neural network; TCAM.

\section{INTRODUCTION}

Unlike legacy routers using distributed routing protocols to decide where to forward the incoming packets. SDN separates the control and data plane. One or more central controllers are responsible for handling the forwarding decision. Switches become simple forwarding devices. SDN controller and applications on top of the controller will make decisions and formulated as rules. After that, the controller will push flow forwarding rule into the switches. In this way, the newly coming packet will be forwarded follow the rule inside of the switch. The separation of the control plane allows smoother control

Permission to make digital or hard copies of all or part of this work for personal or classroom use is granted without fee provided that copies are not made or distributed for profit or commercial advantage and that copies bear this notice and the full citation on the first page. Copyrights for components of this work owned by others than ACM must be honored. Abstracting with credit is permitted. To copy otherwise, or republish, to post on servers or to redistribute to lists, requires prior specific permission and/or a fee.

Request permissions from Permissions@acm.org.

ICIT 2019, December 20-23,2019, Shanghai, China

(C) 2019 Association for Computing Machinery.

ACM ISBN 978-1-4503-7663-1/19/12 ...\$15.00

DOI: https://doi.org/10.1145/3377170.3377218 routing.

Compared to legacy networks, the traffic flows in HPC center is more complicated. Moreover, In SDN switches, rules can be more intricate since SDN switches can match more than 12tuple features [1]. SDN switches use Ternary ContentAddressable Memory(TCAM) [2] as the storage device. The advantage of TCAM is it can perform flow match in $\mathrm{O}(1)$ time complexity [2]. The drawback of TCAM is its cost. This kind of memory is expensive [4] and energy- hungry [5] and thus limits the capacity of the flow table. The actual flow rules in big data center range from 750 to 4000 [6]. As the technology developed, larger TCAM will be applied to SDN switches, but the trade-off between cost and power will still be a big problem which should be concerned. Thus, the actuary of the SDN switch flow table match has become a matter of significant research interest in this area. Several different approaches to managing the TCAM space have been proposed. The first primary method aims at rule replacement [7]-[10]. Another solution focused on flow table rule caching [11]-[13]. We will discuss and compare those two solutions and figure out their advantages and drawbacks.

Intuitively, reduce the total number of rules could improve the TCAM hit rate dramatically. Which means we could replace the fine-grained rule with more general ones by using rule compression. [7] proposed a dynamic flow aggregation procedure. This dynamic approach is based on the network path of the flows. [14] proposed a new framework MINNIE, which use wild-card rules and shortest algorithm to handle the workload balancing. In MINNIE, a hardware-software based hybrid model containing a commercial switch has been discussed. In their experiment result, the factor delay for the first 20 packets increased by 20 percent. In [10], FFTA (Fast Flow Table Aggregation) was proposed, which applied to non-prefix-based rules. However, the ability to support fine- grained rule matching is one of the essential features of SDN. We need to consider is it worth to sacrifice the gratuity to achieve high hit ratio.

Due to the limitations of rule replacement, rule caching method has been studied in recent years. In [11], [12], the author increased the flow table capacity by using a hardware-software switch. They were using a more complex rule caching method called CacheFlow, which construct a dependency tree and will keep more popular rules in the flow table. [13] proposed CRAFT which using a two-stage pipeline to avoid long dependency tree in the network. They split the large flow rules into small sub-rules with high cache hit ratio.

In regards with modern cache manage methods, many wellknown traditional cache replacement policies are still widely using. First in first out FIFO [15] is the most common replacement policy to enable the rule table update. When the 
cache is full, the first coming rule stored in the cache will be evicted first. However, in SDN network environment, due to the high flow rule updating frequency and some of the rules have limited lifetime. FIFO usually isn't an excellent way to do TCAM update. Least Recently Used(LRU) [15] could have better performance in some scenario. Unfortunately, in some particular case, the cache hit ratio in LRU cache is still not acceptable. As well, both FIFO and LRU can only replace one flow rule at one time, which is not enough in the network with a large amount of traffic.

In this paper, we are focusing on the rule table caching method. A prefetching algorithm based on rule correlation alone with time was introduced in [7]. However, it only considered the relationship between the previous flows and future flows, then update the flow rules of every switch in that traffic flow. They did not make full use of the correlation inside of the traffic flow. In our work, we think that flow traffic in an SDN switch is following some specific patterns. We can use these patterns to predict the following flow rules which will be used in the future. Moreover, we find that the flow rules update sequence is similar to the famous speech prediction [16] problem in NLP. Based on that, LSTM [17] is a perfect model to learn the pattern, then to predict the upcoming flow rules in our scenario. From our preliminary results, we find in traces with some internal pattern, LSTM model-based prefetching application can achieve a higher cache hit ratio than LRU and FIFO.

The main contributions of this paper are summarized as follows:

- We analyzed the existing rule aggregation and rule replacement methods for improving SDN switches rule table hit rate. This problem is essential and challenging. The tradeoff between cost and efficiency should be taken into consideration.

- We propose an LSTM based prefetching algorithm to predict the upcoming rules in HPC network. We learn the pattern of the flow rule trace and then generate the possible future rules. To our best knowledge, it is the first work using LSTM model to solve the SDN switch.

- We generated synthetic trace based on the real-world rules to test our method. The preliminary result shows our RNN model can increase the cache hit ratio compare to LRU and FIFO cache.

The remainder of the paper is organized as follows. Section II provides the background of TCAM and LSTM. Section III present the proposed classification method to classify the rule trace and the optimized LSTM model to fit the SDN based HPC workload. Section IV are related works. Section V described the experiment setup and result. The last section includes the conclusion and possible future work.

\section{BACKGROUND}

In this section, we will briefly introduce the SDN rule matching problem and fundamental about LSTM.

\subsection{SDN Flow Table \& TCAM}

Content-addressable memory(CAM) is often compared with Random-addressable memory(RAM). Unlike RAM, the operating system can search CAM by matching the content itself. Due to the better parallel performance. CAM is much faster than RAM. Ternary content-addressable mem- ory(TCAM) can finish searching content in a single clock cycle. The term ternary in TCAM represents the third state $X$ beside 0 , and 1 . $X$ here represent "wildcard". High- speed network routers and switches widely apply TCAM as route lookup memory, to increase the speed of the packet forwarding.

High dimensional rule matching is one of the essential features of SDN rule table. Unlike the legacy switches only use the IP address to decide the packet forwarding path.

SDN flow table can do more than 12 tuple match. Figure 1 provides an example of SDN flow table using TCAM as memory. Each packet has a header file contains the in port number, Ethernet source address, VLAN id, etc. When a new packet coming, SDN switches will check the packet's header file and find one or more matched rule in the flow table. The packet will be forward or drop based on the matched rule with the highest priority. If not match found, SDN switch will send the packet to the controller. The controller then decides where to forward the packet. In this scenario, communication between switch and controller will bring significant overhead, which will decrease the efficiency of the entire network.

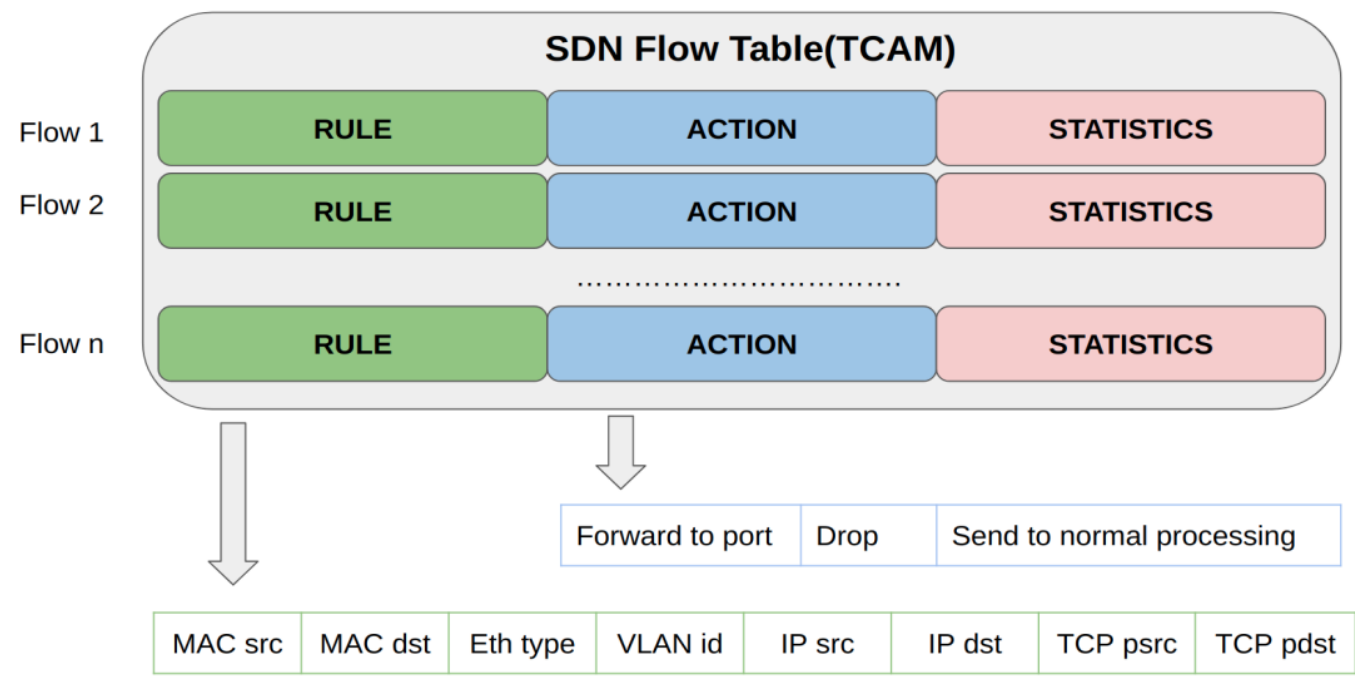

Figure 1.SDN flow table 


\subsection{Prefetching}

Prefetching is a widely-applied technique in computer system design, especially in the CPU cache. The system with prefetching mechanism can pre-load the content to CPU cache or memory buffer, before the real request, and then reduce the access overhead. The efficiency of the prefetching is highly depended on the accuracy of the prefetching algorithm. A good algorithm can predict the content expected to be needed soon. The property and function of SDN switch flow table is quite similar to CPU cache or DRAM buffer. However, unlike cache and DRAM buffer store data in the user system, SDN flow table store rules of the network. Naturallyit might be possible to apply prefetching techniques to SDN to improve the flow table update efficiency.

\section{SYSTEM DESIGN}

We will discuss how we design to improve the performance of the LSTM based prefetching algorithm in this section. We will focus on the design by addressing the following.

\subsection{Using LSTM for Flow Rules Prediction}

LSTM has been widely used in numerous real-world application, such as text tagging, music generation, and chat bot [18]. Using LSTM model to dealing prediction and other real-world problems involves two steps: training and testing. Offline and online training are two different ways applying to various applications. Online training requires retraining the model by using new data in the new time window. When training process finishes, the "model" is actually stored as some vector of parameters, which will be prepared for use in the inference/testing phase.

SDN flow table rule prediction is essentially a time series problem. The workload trace has complex non-linear relationships. Compared with SDN workflow, DRAM activity is $\mathrm{PC}$ is relatively clean and with more internal correlation. This propriety makes CPU cache prefetching relatively an easier task. On the contrary, real-world SDN network traces often involve numerous noises from different workloads, especially in some busy environment like HPC. Due to the specific internal data structures of various kinds of groups, there exists repeated access patterns in the workflow. However, application in the data center generates multiple flows con- currently, which interweaves the traces of individual threads stochastically. In terms of each flow, the amount of noise substantially exceeds that of its original flow. Therefore, it is challenging to discover access patterns from an entangled trace. In Figure 2, workflow A and B both have a simple and straightforward internal pattern. Flow A follows a sequential rule matching pattern. In contrary, flow B has reversed sequential matching pattern. Predict the upcoming rule from $\mathrm{A}$ or $\mathrm{B}$ its not hard. However, if $\mathrm{A}$ and $\mathrm{B}$ are randomly entangled with each other, the pattern of both $\mathrm{A}$ and B will be interleaved, which makes it extremely hard to predict. Fortunately, LSTM network has the ability to handle the no-linearity and noise. Our objective is using deep learning techniques to construct the complex high dimensional nonlinear function, and use it to carry out of the prediction of upcoming flow rules. While the sequence entanglement is one of the main problems in flow rule prediction task, we essentially apply clustering LSTM [12] as our main prediction component. The prediction problem is formulated as a task to predict the rules used by the upcoming packets based on the previous ones.

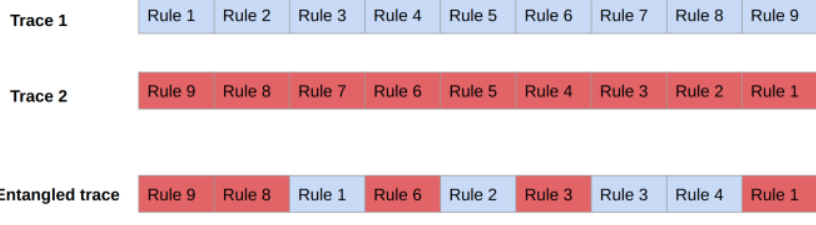

Figure 2. Single trace \& entangled trace

\subsection{LSTM Model Architecture}

To predict future comping packets, we implement an LSTM model which consist of 2 parts, the offline training part, and the online inference part. Webuild the LSTM model based on PyTorch [19] framework. We divided the trace data into training and validation sets. Training data consist of $70 \%$ of the total trace, which is used to optimizing the parameters in our LSTM, while the other $30 \%$ validation set is used to test the performance of the model.

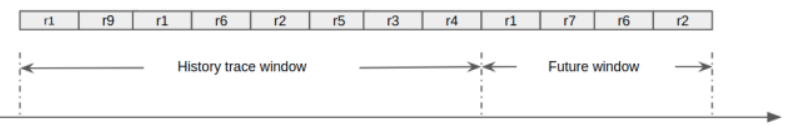

Figure 3. The input and output of SDN rule prefetching model

Since the flow rule prediction model is similar to music prediction models which infer the future from past time series sequences. We bring the idea of music/text generation LSTM model structure to build the SDN rule prediction model. As shown in Figure 3, a sliding window with adjustable length containing the history flow trace, are fit as model inputs. Due to the large rule space in real SDN networks. We use an embedding level before the LSTM layer to map the high dimensional one-hot representation of rules into low dimensional vectors. In LSTM layers, 128 hidden cells are deployed inside of each LSTM cell. The LSTM hidden layers structure are formulated as the following functions:

$$
\begin{gathered}
i_{t}=\sigma\left(W_{i i} x_{t}+b_{i i}+W_{h i} h_{t-1}+b_{h i}\right) \\
f_{t}=\sigma\left(W_{i f} x_{t}+b_{i f}+W_{h f} h_{t-1}+b_{h f}\right) \\
g_{t}=\tanh \left(W_{i g} x_{t}+b_{i g}+W_{h g} h_{t-1}+b_{h g}\right) \\
o_{t}=\sigma\left(W_{i o} x_{t}+b_{i o}+W_{h o} h_{t-1}+b_{h o}\right) \\
c_{t}=f_{t} * c_{t-1}+i_{t} * g_{t} \\
h_{t}=o_{t} * \tanh \left(c_{t}\right)
\end{gathered}
$$

where $\sigma$ is the sigmoid activation function. $h_{t}$ and $t_{1}$ stands for the hidden state at time $\mathrm{t}$ and $\mathrm{t}-1$ respectively. $f_{t}, g_{t}, o_{t}$ represent the forget, cell, and output gates. * means Hadamard product.

The number of LSTM cells can be various depend on the length of the time window. In addition, the outputs of LSTM hidden layers are will be used as input to another liner fully connected layer. We choose $\mathrm{m}$ number of flows as history window length and predict the upcoming $\mathrm{n}$ possible flows. We tested different $\mathrm{m}$ and $\mathrm{n}$, and find out that when $\mathrm{m}$ equals to 10 produce the best result since the training process is handled by GPU, located outside of the SDN application. So we don't need to worry about the training time. When the training process finished, SDN prefetching application, on top of the controller, utilize the well-trained parameter to predict the upcoming rules. 


\subsection{SDN Application Design}

To improve the hit ratio in TCAM, prefetching has to happen before the new packet coming. The workflow of this application is as follows: Before new packet comes in, the application will call southbound APIs, the LSTM model start to predict the next $\mathrm{n}$ possible rules, $\mathrm{n}$ is a parameter can be configured by the user. Then updating APIs will push those rules into the SDN switch. A pre-active application is proposed so far. We assume the pattern of the flow will not change by time in the preliminary experiment. Hence, the prediction API will be called in a fixed time period. The architecture of the prefetching application is shown in Figure 4. The SDN application is on top of the SDN controller. Both training and prediction will execute outside of the application space.

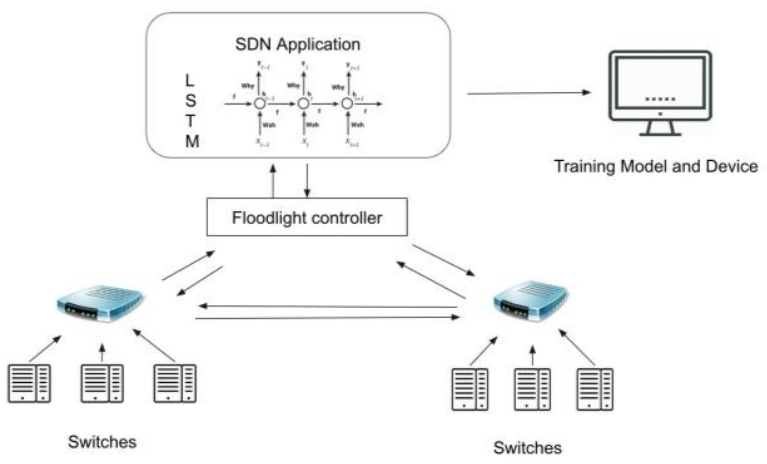

Figure 4. Application structure

\section{EXPERIMENTAL SETUP}

We simulate and generate the network trace by using Python3.4. Then we test the LSTM rule prefetching model by real-world rules provided by REANNZ research and education network [21]. Since the policy only provides 460 OpenFlow 1.0 rules. But without traffic trace, we generate simulated trace based on Zip(f) distribution with length 100000 . Two types of traffic trace have been tested, the clean periodical traffic and the other one with $30 \%$ noises. Noises are generated follow Gaussian distribution. We simulate HPC networks using MININET [19], and use Floodlight [20] as the main controller to design the application. Besides, we implemented LRU and FIFO cache using Python and set LRU and FIFO without prefetching as the baseline.

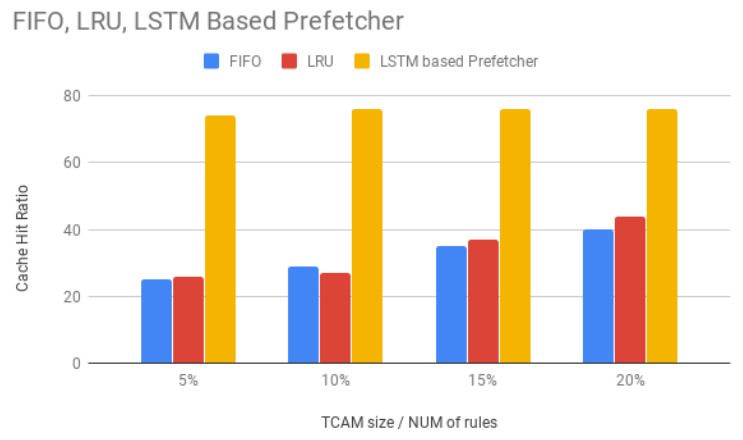

Figure 5. Periodical clean traffic trace

\section{EXPERIMENTAL RESULTS}

Both clean periodical trace and trace with noises are split into training and testing data sets. We use the trained models to do the rule prefetch and study the cache hit ratio, and then compare the performance with the traditional FIFO and LRU cache with out any replacement policy. We compare the cache hit ratio of the two cache replacement policies, and use different cache size to conduct sensitivity study.

Figure 4 shows the cache hit ratio using different configurations. We use the synthetic data with simple pattern first. TCAM size is equals to $5 \%, 10 \%, 15 \%, 20 \%$ of the total number of rules respectively. From Figure 5, due to the memorable ability of LSTM, the SDN rule table prefetcher works surprising well on clean periodical traffic pattern. The average cache hit ratio increased by $2 \mathrm{x}$ compare with pure LRU and FIFO. LRU and FIFO achieved similar hit ratio due to the internal periodical pattern.

From Figure 6, the performance of the LSTM based prefetcher decreased significantly due to the high noise ratio of the trace. As we discussed in Section 3, even random entangle the simple sequential traces will make the pattern extremely hard to find. We tried to classify the entangled trace first, then apply the LSTM model to do prefetching. However, the converge rate is still not promising. We think the high noise ratio will cause the LSTM model hard to find the global minimum. It still remains a lot of challenges to predict the trace with a high noise ratio. We will try to improve our model to solve this problem in the future works.

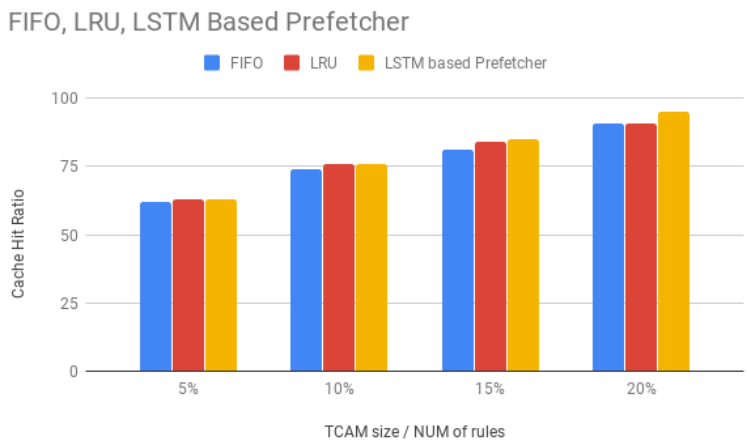

Figure 6. Trace with noise

\section{CONCLUSIONS}

As we know, this is the first work using deep learning to optimize the flow table hit ratio problem in SDN switches. From the preliminary result, we surprisingly find that the RNN based model can have even better performance than traditional FIFO and LRU. Although we only use synthetic data for training and testing. The result still shows it is a possible way to apply deep learning method for the SDN network and architecture optimization problem in the future.

We propose to explore several future works for the pro- posed model. First, a reactive SDN application to improve the prediction accuracy by training online is desirable. In this way, the model can be updated attentively to a dynamic network environment. The reactive application could trigger the retraining process by monitoring the workflow in SDN network, and start the training when the hit ratio drops below some predefined threshold. Second, we will conduct several experiments based on the real-world trace, from different data centers. Third, we will test out different models on similar flow traces at a larger workload space. 


\section{ACKNOWLEDGMENT}

This project is supported in part by the US National Science Foundation Grant CCF-1527249, 1717388, and 1907765, and US Army/DURIP program W911NF-17-1-0208.

\section{REFERENCES}

[1] McKeown, N., Anderson, T., Balakrishnan, H., Parulkar, G., Peterson, L., Rexford, J., Shenker, S. and Turner, J., 2008. OpenFlow: enabling innovation in campus networks. ACM SIGCOMM Computer Com- munication Review, 38(2), pp.69-74.

[2] Salisbury, B., 2012. TCAMs and OpenFlow-what every SDN practi- tioner must know. See http://tinyurl. com/kjy99uw.

[3] Guo, Z., Liu, R., Xu, Y., Gushchin, A., Walid, A. and Chao, H.J., 2017. STAR: Preventing flow-table overflow in software-defined networks. Computer Networks, 125, pp.15-25.

[4] SDN sy stem performance. See http://pica8.org/blogs/?p=201, 2012.

[5] Spitznagel, E., Taylor, D. and Turner, J., 2003, November. Packet classification using extended TCAMs. In 11th IEEE International Conference on Network Protocols, 2003. Proceedings. (pp. 120-131). IEEE.

[6] Stephens, B., Cox, A., Felter, W., Dixon, C. and Carter, J., 2012, December. PAST: Scalable Ethernet for data centers. In Proceedings of the 8th international conference on Emerging networking experiments and technologies (pp.4960). ACM

[7] Li, H., Guo, S., Wu, C. and Li, J., 2015, June. FDRC: Flowdriven rule caching optimization in software defined networking. In 2015 IEEE International Conference on Communications (ICC) (pp. 5777-5782). IEEE.

[8] Mimidis, A., Caba, C. and Soler, J., 2016, June. Dynamic aggregation of traffic flows in SDN: Applied to backhaul networks. In NetSoft Conference and Workshops (NetSoft), 2016 IEEE (pp. 136-140). IEEE.

[9] Das, S., Yiakoumis, Y., Parulkar, G., McKeown, N., Singh, P., Getachew, D. and Desai, P.D., 2011, March. Applicationaware aggre- gation and traffic engineering in a converged packet-circuit network. In National Fiber Optic Engineers Conference (p. NThD3). Optical Society of America.

[10] Luo, S. and Yu, H., 2014, August. Fast incremental flow table aggre- gation in SDN. In Computer Communication and Networks (ICCCN), 2014 23rd International Conference on (pp. 1-8). IEEE.

[11] Katta, N., Alipourfard, O., Rexford, J. and Walker, D., 2014, August. Infinite cacheflow in software-defined networks. In Proceedings of the third workshop on Hot topics in software defined networking (pp. 175-180). ACM.

[12] Katta, N., Alipourfard, O., Rexford, J. and Walker, D., 2016, March. Cacheflow: Dependency-aware rule-caching for software-defined net- works. In Proceedings of the Symposium on SDN Research (p. 6). ACM.

[13] Li, X. and Xie, W., 2017, July. CRAFT: A Cache Reduction Ar- chitecture for Flow Tables in SoftwareDefined Networks. In 2017 IEEE Symposium on Computers and Communications (ISCC) (pp.967-972). IEEE.

[14] Rifai, M., Huin, N., Caillouet, C., Giroire, F., LopezPacheco, D., Moulierac, J. and Urvoy-Keller, G., 2015, December. Too many SDN rules? Compress them with MINNIE. In 2015 IEEE Global Communications Conference (GLOBECOM) (pp. 1-7). IEEE.

[15] A. and Towsley, D., 1990. An approximate analy sis of the LRU and FIFO buffer replacement schemes (Vol. 18, No. 1, pp. 143-152). ACM.

[16] Markel, J.D. and Gray, A.J., 2013. Linear prediction of speech (Vol. 12). Springer Science \& Business Media.

[17] Sak, H., Senior, A. and Beaufays, F., 2014. Long shortterm mem- ory recurrent neural network architectures for large scale acoustic modeling. In Fifteenth annual conference of the international speech communication association.

[18] http://karpathy.github.io/2015/05/21/rnn-effectiveness/ The Unreason- able Effectiveness of Recurrent Neural Networks

[19] MININET http://mininet.org/

[20] Floodlight OpenFlow Controller http://www.projectfloodlight.org/floodlight/

[21] "REANZZ"http://reannz.co.nz/ 